\title{
Versican silencing improves the antitumor efficacy of endostatin by alleviating its induced inflammatory and immunosuppressive changes in the tumor microenvironment
}

\author{
ZI WANG $^{1,2^{*}}$, ZHIXI LI $^{1 *}$, YUYI WANG ${ }^{1 *}$, DAN CAO $^{1}$, XIA WANG $^{1}$, MING JIANG ${ }^{1}$,

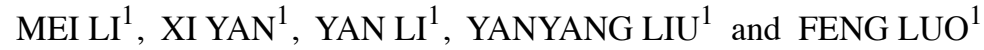 \\ ${ }^{1}$ Department of Medical Oncology, Cancer Center and State Key Laboratory of Biotherapy, \\ West China Hospital of Sichuan University, Chengdu, Sichuan 61004; ${ }^{2}$ Department of Oncology, \\ Guizhou Provincial People's Hospital, Guiyang, Guizhou 550002, P.R. China
}

Received January 22, 2015; Accepted March 17, 2015

DOI: 10.3892/or.2015.3903

\begin{abstract}
The systematic application of antiangiogenic therapy remains an issue of concern, mainly due to the hypoxic and inflammatory changes in the tumor microenvironment elicited by antiangiogenic therapy. Versican, a 'bridge' connecting inflammation with tumor progression as well as playing a central role in the generation of an inflammatory tumor microenvironment is a promising candidate for the intervention of inflammatory changes in the tumor microenvironment elicited by antiangiogenic therapy. To examine this hypothesis, a short-hairpin RNA targeting versican (shVCAN) was designed and shVCAN stable-transfected B16F1 and Lewis lung carcinoma (LLC) cell lines were established. Simultaneously, the established B16F1 and LLC tumor models were used to investigate the effect of versican silencing on the tumor burden of mice. The results showed that, versican silencing exerted an inhibitory effect on the proliferative and migratory ability of B16F1 cells, but did not affect LLC cells. Endostatin exhibited modest inhibition of tumor growth in tumor-bearing mice. Versican silencing alone effectively suppressed orthotopic tumor growth and significantly prolonged survival time of mice more effectively when combined with endostatin. Endostatin elicited inflammatory and immunosuppressive changes in the tumor microenvironment, including an accumulation of myeloid-derived suppressor cells (MDSCs), tumor-associated macrophages (TAMs) and inflammatory cytokines. In addition, NF- $\mathrm{BB}$ and HIF-1 $\alpha$ were overexpressed in the tumor. Versican silencing improved the antitumor
\end{abstract}

Correspondence to: Dr Feng Luo, Department of Medical Oncology, Cancer Center and State Key Laboratory of Biotherapy, West China Hospital of Sichuan University, 37 Guoxue Xiang Street, Chengdu, Sichuan 61004, P.R. China

E-mail: luofeng@medmail.com.cn

*Contributed equally

Key words: versican, endostatin, antiangiogenic therapy, tumor microenvironment efficacy of endostatin by alleviating its induced changes in the tumor microenvironment. Thus, versican silencing in the tumor microenvironment offers a promising approach to reverse the tumor refractoriness to antiangiogenic therapies.

\section{Introduction}

Antiangiogenic therapy is considered significant for the treatment of cancer (1). However, accumulating evidence suggests that antiangiogenic therapy shows transient antitumor activity and enhances tumor invasiveness and metastasis $(2,3)$. The complex changes in the tumor microenvironment elicited by antiangiogenic therapy, which result in a hypoxic and inflammatory microenvironment, are the main cause of tumor refractoriness. Notably, numerous tumor-associated inflammatory cells and their cytokines, e.g., $\mathrm{CD} 11 \mathrm{~b}^{+} \mathrm{Gr} 1^{+}$ myeloid-derived suppressor cells (MDSCs), tumor-associated macrophages (TAMs), granulocyte colony-stimulating factor (G-CSF) and tumor necrosis factor- $\alpha$ (TNF- $\alpha$ ), are recruited into the tumor microenvironment $(4,5)$. Therefore, remission or resolution of the inflammatory tumor microenvironment is crucial for improving the efficacy of antiangiogenic therapies.

Versican, a versatile extracellular matrix proteoglycan present in a variety of tissues and commonly overexpressed in tumor stroma and cancer cells (6), plays a key role in cancer development and progression by contributing to cell adhesion, migration, angiogenesis and the formation of an inflammatory tumor microenvironment (7). By activating multiple types of inflammatory cells through the Toll-like receptor 2 (TLR-2) and then eliciting the production of many proinflammatory cytokines, versican strongly enhances tumor progression (8). Additionally, the inflammatory and tumor cells enhance versican expression, which in turn induces the secretion cascade of inflammatory cytokines to generate an inflammatory microenvironment that provides permissive conditions for tumor progression and metastases (9).

Endostatin, a broad-spectrum endogenous angiogenesis inhibitor, suppresses tumor growth mainly by selectively blocking the binding of vascular endothelial growth factor (VEGF) to endothelial cells (ECs) to inhibit the 
VEGF-stimulated proliferation, migration and tube formation of ECs $(10,11)$. Several lines of direct and indirect evidence indicate that endostatin has caused a significant reduction in microvessel density and resulted in the inhibition of tumor growth. However, the antitumor efficacy of endostatin alone is of short duration, and confers no significant survival benefit to tumor bearing mice (12-14). Findings of a recent study showed that versican, $\mathrm{NF}-\kappa \mathrm{B}$ and HIF-1 $\alpha$, which are associated with inflammatory and hypoxic changes in the tumor microenvironment, were found to be overexpressed in tumor tissues from animal models refractory to endostatin treatment. Additionally, MDSCs and inflammatory cytokines were largely recruited into the peripheral blood and the tumor microenvironment (15). However, the role of versican and tumor-associated inflammatory cells in the tumor microenvironment changes caused by endostatin administration is not entirely clear, and the connection of versican with tumor-associated inflammatory cells, and with other inflammatory and immune regulators in the tumor microenvironment, remains to be elucidated. Accordingly, we hypothesized that versican, a 'bridge' connecting inflammation with tumor progression as well as playing a central role in the generation of inflammatory tumor microenvironment is a promising candidate for the intervention of inflammatory changes in the tumor microenvironment elicited by antiangiogenic therapy.

Thus, we designed a short-hairpin (sh) RNA targeting versican and assessed the effects of versican silencing on the bioactivity of B16F1 and Lewis lung carcinoma (LLC) cell lines. At the same time, the established B16F1 and LLC tumor models were used to investigate the effect of versican silencing combined with endostatin on the tumor burden of mice. Furthermore, we studied the effect of versican on the changes in the tumor microenvironment elicited by endostatin, and examined the related mechanisms.

\section{Materials and methods}

Cell culture. LLC and B16F1 melanoma cell lines were obtained from the American Type Culture Collection (ATCC; Manassas, VA, USA). The cells were cultured in Dulbecco's modified Eagle's medium (Gibco-BRL, Grand Island, NY, USA) supplemented with $10 \%$ calf serum and $1 \%$ penicillin/ streptomycin, and maintained in a humidified $5 \% \mathrm{CO}_{2}$ atmosphere incubator at $37^{\circ} \mathrm{C}$.

Knockdown of versican in LLC and B16F1 cells. Short-hairpin RNAs (shRNA) targeting mouse versican (V1 isoform) were designed and cloned into the pcDNA6.2-GW/EmGFP-miR vector (Invitrogen, Shanghai, China), and transfected into LLC and B16F1 cells by Lipofectamine 2000 (Invitrogen) according to the manufacturer's instructions. The cells were then selected in $8 \mu \mathrm{g} / \mathrm{ml}$ Blasticidin $\mathrm{S} \mathrm{HCl}$ (Invitrogen), sorted for green fluorescent protein (GFP) expression and cloned. The targeting sequence included 5'-GTACACAGTTGATGAAATAC-3'. The pcDNA6.2-GW/EmGFP-miR-neg (shC) plasmid served as a negative control (Invitrogen).

Groups. Six groups of each model were analyzed in the present study: normal saline (B16F1-NS and LLC-NS), normal saline + pcDNA6.2-GW/EmGFP-miR-neg (B16F1/shC-NS,
LLC/shC-NS), normal saline + pcDNA6.2-GW/EmGFPmiR-versican (B16F1/shVCAN-NS, LLC/shVCAN-NS), endostatin (B16F1-ES, LLC-ES), endostatin + pcDNA6.2GW/EmGFP-miR-neg (B16F1/shC-ES, LLC/shC-ES) and endostatin + pcDNA6.2-GW/EmGFP-miR-versican (B16F1/shVCAN-ES, LLC/shVCAN-ES).

Western blot analysis. Equal amounts of protein were separated by $8-12 \%$ SDS-PAGE and transferred to polyvinylidene fluoride (PVDF) membranes (Millipore, Shanghai, China) by electroblotting. The membranes were probed with specific antibodies including HIF-1 $\alpha$ (1:100; ab113642), versican (1:200; ab19345) (both from Abcam, Cambridge, UK) or NF-кB (1:100; no. 8242S; CST, Boston, MA, USA). Blots were developed with horseradish peroxidase (HRP)-conjugated secondary antibodies and chemiluminescent substrate on Kodak X-ray film.

Cell proliferation, Transwell migration and invasion assays. Cells were seeded at a density of $1 \times 10^{3}-3 \times 10^{3}$ cells/well on 96-well plates, cultured for $72 \mathrm{~h}$, and subjected to a CCK- 8 colorimetric assay (Dojindo, Shanghai, China), according to the manufacturer's instructions. The migration and invasive ability of cells was determined using the Matrigel (BD Biosciences)-coated 24-well Transwell chambers (Corning Costar, Beijing, China). The cells $\left(1 \times 10^{5}\right)$ were seeded in the top chamber and incubated for 24 or $48 \mathrm{~h}$. The migrating or invading cells were counted using a light microscope (Olympus UIS2; magnification, x20, three random fields/well were analyzed by ImageJ).

Tumor models and treatment protocol. C57BL/6 mice were subcutaneously implanted with $100 \mathrm{ml}$ solution containing $3-5 \times 10^{5}$ cells in the right mid-dorsal flank. The tumor volume $\left(\mathrm{mm}^{3}\right)$ was calculated as length $\mathrm{x}$ width ${ }^{2} / 2(16)$. The time when the tumors reached volumes of $10-50 \mathrm{~mm}^{3}$ was designated as day 0 , after which normal saline or endostatin [3 mg/ $\mathrm{kg}(15)$; Simcere-Medgenn Bio-Pharmaceutical Co., Ltd. Shandong, China] was administered daily i.v. (by caudal vein injection) for 9 days to the tumor-bearing mice. On days $0,3,6$ and 9 after initiation of treatment, the tumor volume was estimated and mice (three from each group) were sacrificed to harvest tumor tissues and blood samples for subsequent analyses (15). Experimental procedures and protocols were approved by the Animal Ethics Committee of Sichuan University.

Flow cytometry. Analyses of the MDSCs and TAMs in the tumor tissue and peripheral blood were conducted as previously described (15). Single-cell suspensions were stained with fluorochrome-labeled antibody-targeting murine CD11b (PE-CY5-labeled), Gr1 (PE-labeled), F4/80 (APC-labeled) or an appropriate isotype control antibody (all from Tianjin Sungene Biotech Co., Ltd.) and were analyzed by flow cytometry (BD FACSCalibur; BD Biosciences).

Luminex xMAP assays. A commercially available mouse cytokine magnetic bead panel kit (Millipore, Billerica, MA, USA) was used to evaluate the cytokine levels in collected mouse serum and tumor tissue. On days 0,3,6 and 9 after initiation of treatment, the serum was collected from nonanticoagulated blood from all mice, and the protein lysate of 
A

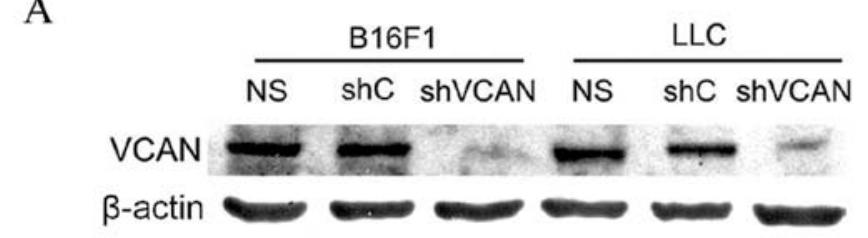

B

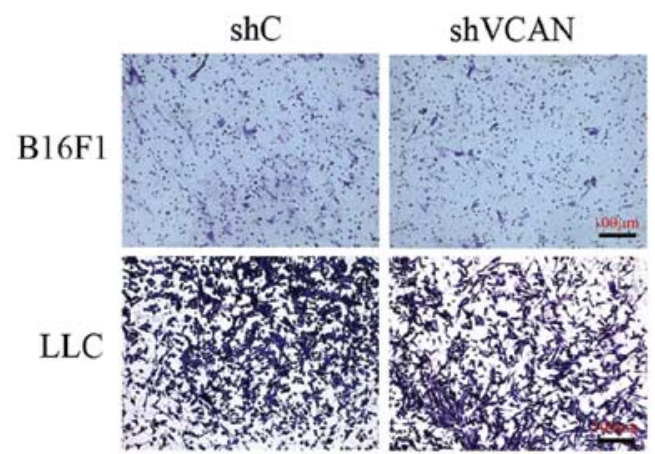

C

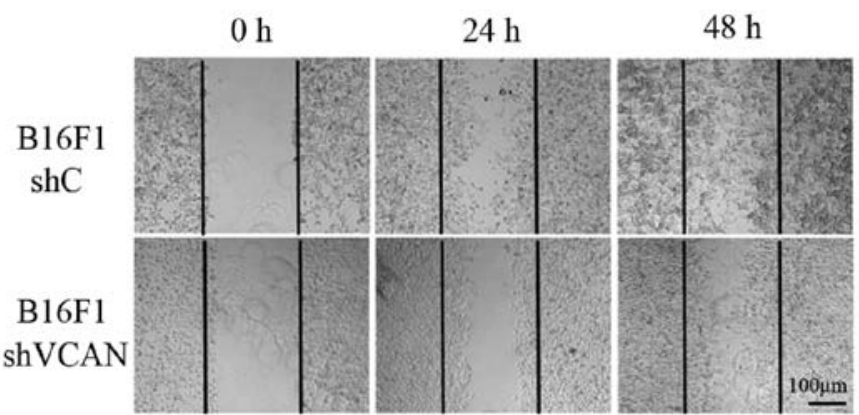

D

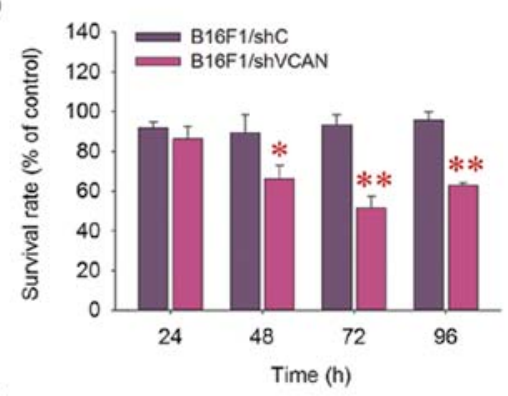

E

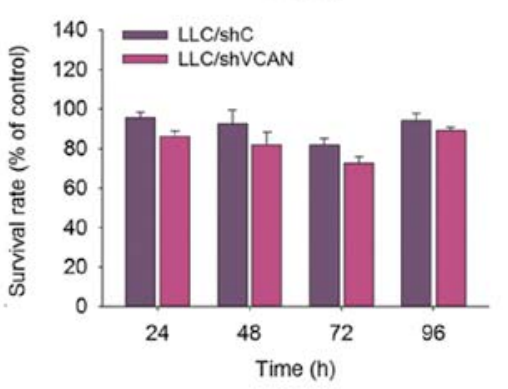

F

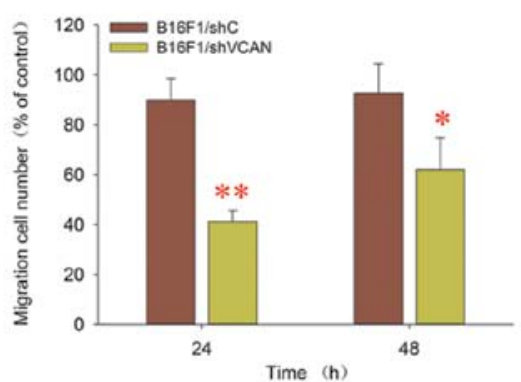

Figure 1. Identification of stable-transfected B16F1 and LLC cell lines and the effects of versican silencing on their bioactivity. (A) Versican silencing was confirmed by western blot analysis. (B) Transwell invasion assays were conducted to evaluate the invasive ability of cells on the effect of versican silencing. $(\mathrm{C}$ and $\mathrm{F}$ ) Wound-healing assays were carried out to investigate the effects of versican silencing on cell migration. (D and $\mathrm{E}$ ) $\mathrm{CCK}-8$ analysis was performed to assess the effects of shRNA-mediated versican silencing on cell proliferation. Original magnification, $\mathrm{x} 200$ for all panels. shVCAN vs. shC, ${ }^{*} \mathrm{P}<0.05,{ }^{* *} \mathrm{P}<0.01$. LLC, Lewis lung carcinoma; shVCAN, short-hairpin RNA targeting versican.

tumor tissue was collected. The cytokines comprising G-CSF, TNF- $\alpha$, IL-6, VEGF and IL-10 were measured according to the manufacturer's instructions (MILLIPLEX ${ }^{\circledR}$ MAP) based on Luminex xMAP technology (Millipore).

Immunohistochemistry. As previously described (15), paraffin-embedded tumor tissues were sectioned (4-5 $\mu \mathrm{m})$, and incubated with the following antibodies: HIF-1 $\alpha$, versican (both from Abcam, Cambridge, UK), NF- $\kappa$ B (CST) and CD31 (BD Biosciences). Randomly chosen fields were photographed at a magnification of $\mathrm{x} 400$ with a microscope (Leica DM2500, Germany). Immunopositive cells and staining intensities were quantified by measuring the pixel area of the positive-stained tissue using Image-Pro Plus 6 software. At least three random fields were evaluated for each section and the averages were compared.

Statistical analysis. Data were presented as means \pm SD. The Statistical Package for the Social Sciences (SPSS) version 19.0 (Chicago, IL, USA) was used for statistical analysis. Statistical significance between the groups was determined using one-way ANOVA, and the Bonferroni method was used to compare multiple means. Differences were considered significant at $\mathrm{P}<0.05$.

\section{Results}

Construction of shVCAN stable-transfected B16F1 and LLC cell lines. We first performed versican knockdown in B16F1 and LLC cells in vitro through the construction of shVCAN stable-transfected cell lines as described in 'Materials and methods'. The overall transfection rates of the stable transfected cell lines were estimated to be $>70 \%$, as confirmed by flow cytometric analysis of GFP (data not shown). Successful silencing of versican expression was confirmed by western blot analysis, which revealed that versican expression was markedly downregulated in shVCAN stable-transfected cell lines (Fig. 1A).

Effects of silencing of versican on the bioactivity of B16F1 and $L L C$ cells in vitro. To assess the potential effects of versican silencing on the bioactivity of shVCAN stable-transfected cell lines. CCK-8 analysis, wound-healing and Transwell invasion assays were performed. The results showed that versican silencing had an inhibitory effect on the proliferative and migratory ability of B16F1 cells, but did not affect the invasion properties of $\mathrm{B} 16 \mathrm{~F} 1$ cells. However, the versican silencing had no effect on LLC cells in terms of the aforementioned bioactivity (Fig. 1B-F). 
A

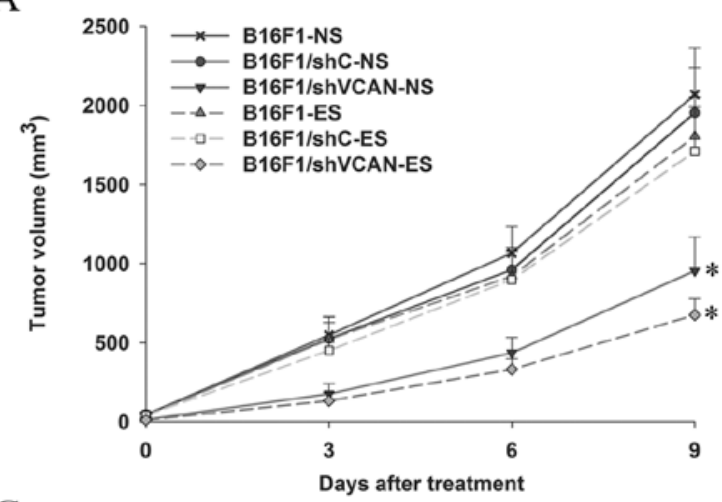

\section{B}

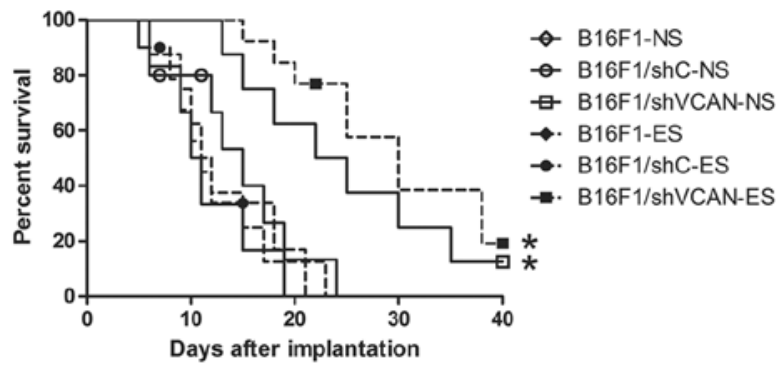

$\mathrm{C}$

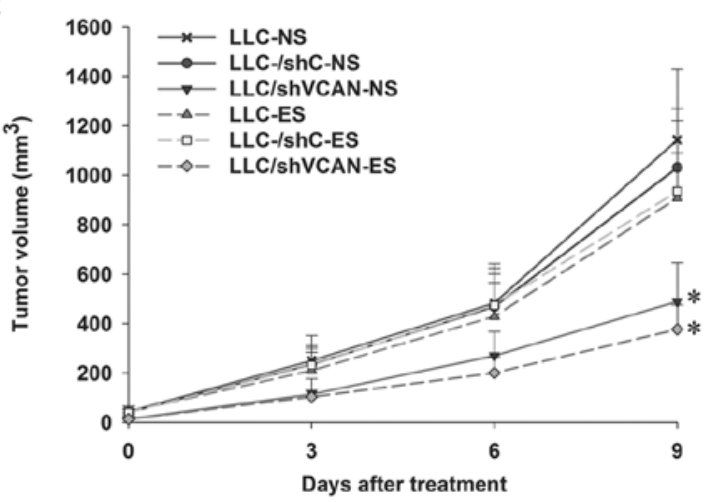

$\mathrm{D}$

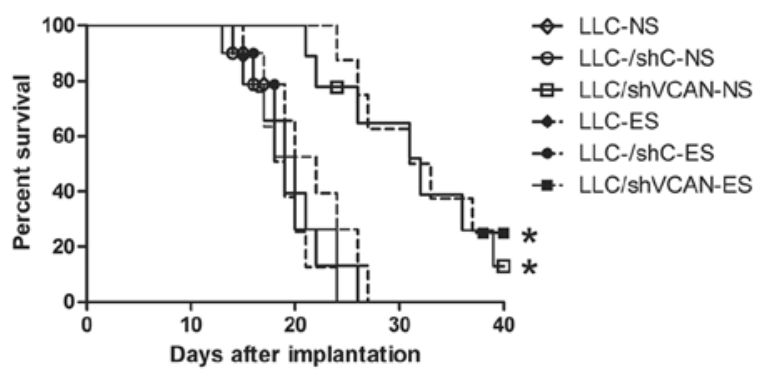

Figure 2. Primary tumor growth and the life-prolonging effect of versican silencing and endostatin in established B16F1 and LLC tumor models. (A and C) The growth curves of B16F1 and LLC tumor xenografts. Tumor volume was estimated every 3 days; $n=6$. Data are presented as mean \pm SD. shVCAN vs. shC, ${ }^{*} \mathrm{P}<0.05,{ }^{* *} \mathrm{P}<0.01$. (B and D) Survival curves of B16F1 and LLC animals. Life-prolonging effect was determined from the survival time (days post-implantation) of the tumor-bearing mice. $\mathrm{n}=10$. shVCAN vs. shC, ${ }^{*} \mathrm{P}<0.05,{ }^{* *} \mathrm{P}<0.01$. LLC, Lewis lung carcinoma; shVCAN, short hairpin RNA targeting versican.

Antitumor efficacy of versican silencing and endostatin in vivo. The established B16F1 and LLC tumor models were used to investigate the effect of combining versican silencing with endostatin on the tumor burden of mice. In the first experiment, the mice were observed for primary tumor growth (Fig. 2A and C). The monotherapy with endostatin showed modest inhibition of tumor growth compared with the NS-treated groups. The shVCAN stable-transfected groups showed significant inhibition of tumor growth compared with the NS-treated and shC stable-transfected control groups (more effectively in shVCAN-ES groups). The second experiment was conducted to examine the life-prolonging effect of the treatments (Fig. 2B and D). All the groups of mice that received NS-treated, shC stable-transfected or endostatin treatment alone died of tumor burden within 24 days (LLC, 27 days) of B16F1 implantation. The shVCAN-transfected groups showed a significantly prolonged survival time. These results indicated that the antitumor efficacy of endostatin was modest. However, versican silencing had a significant antitumor effect that was more effective when combined with endostatin.

Versican silencing reduces the accumulation of MDSCs and TAMs elicited by endostatin. To test the hypothesis that versican silencing in the tumor microenvironment reduces the tumor refractoriness to endostatin by reducing the recruitment of tumor-associated inflammatory cells, we examined the presence of MDSCs in the tumor tissue and peripheral blood, and TAMs in the tumor tissue, by flow cytometry. Figs. 3 and 4 show that MDSCs in the tumor tissue and periph- eral blood increased gradually with the duration of endostatin treatment (on day 9 after treatment, $\mathrm{P}<0.05$ ). However, a clear reduction in MDSCs was found in all shVCAN stable-transfected groups, as compared with shC stable-transfected and untransfected models (on day 3-9 after treatment, $\mathrm{P}<0.05$ ). Notably, on day 3 after treatment, a reduction in MDSCs in the tumor tissue was observed in the LLC/shVCAN-ES group, as compared with the LLC/shVCAN-NS group. As shown in Fig. 5, a modest reduction in TAMs in the tumor tissue of $\mathrm{B} 16 \mathrm{~F} 1 / \mathrm{shVCAN}-\mathrm{ES}$ group was found, as compared with the B16F1-ES group (on day 6 after treatment, $\mathrm{P}<0.05$ ). However, a reduction in TAMs in the tumor tissue of LLC tumor models was found in all shVCAN stable-transfected groups only on day 0 after treatment, as compared with the shC stable-transfected and untransfected groups $(\mathrm{P}<0.05)$. Taken together, these results indicated that MDSCs were largely recruited into the peripheral blood and the tumor microenvironment of tumor models after endostatin treatment; however, versican silencing reduced the MDSCs accumulation. The recruitment of TAMs in tumor tissue after endostatin treatment was found only on day 9 after treatment. Versican silencing reduced the number of TAMs in the tumor tissue on day 6 after treatment in B16F1 tumor models (and on day 0 in LLC tumor models).

Versican silencing reduces the accumulation of tumor-associated inflammatory cytokines elicited by endostatin. To determine the inflammatory changes in the tumor microenvironment elicited by endostatin, Luminex XMAP assays were conducted to examine the level of inflammatory cytokines, 
Tumor tissue
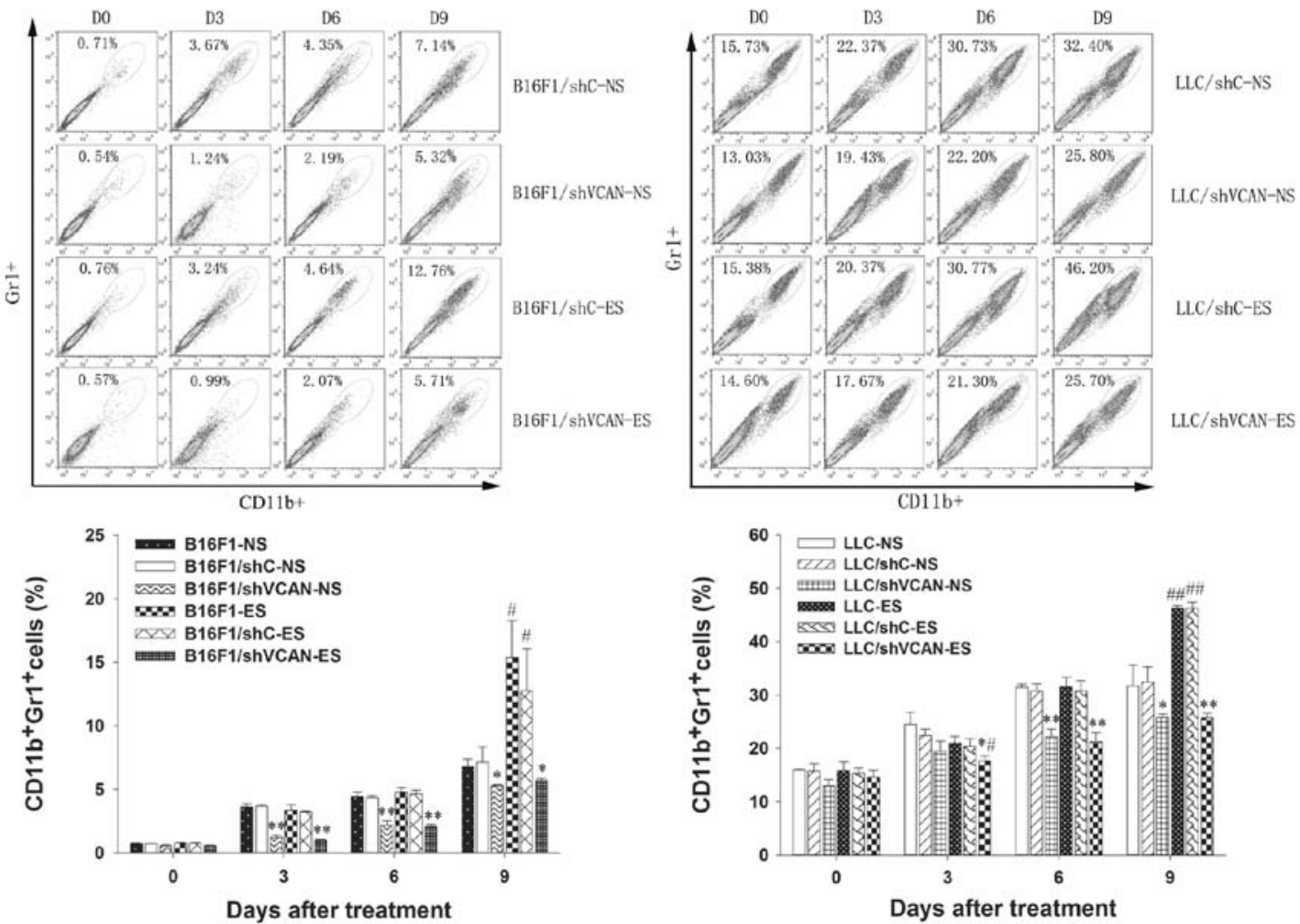

Figure 3. Versican silencing reduces the accumulation of MDSCs elicited by endostatin in tumor tissue. On days 0,3,6 and 9 after the initiation of endostatin treatment, flow cytometry was performed to determine the presence of MDSCs in the tumor tissue of B16F1 and LLC tumor-bearing mice. $\mathrm{n}=3$. shVCAN vs. shC, ${ }^{*} \mathrm{P}<0.05,{ }^{* *} \mathrm{P}<0.01$. ES vs. NS, ${ }^{\#} \mathrm{P}<0.05,{ }^{\# /} \mathrm{P}<0.01$. MDSCs, myeloid-derived suppressor cells; shVCAN, short-hairpin RNA targeting versican.
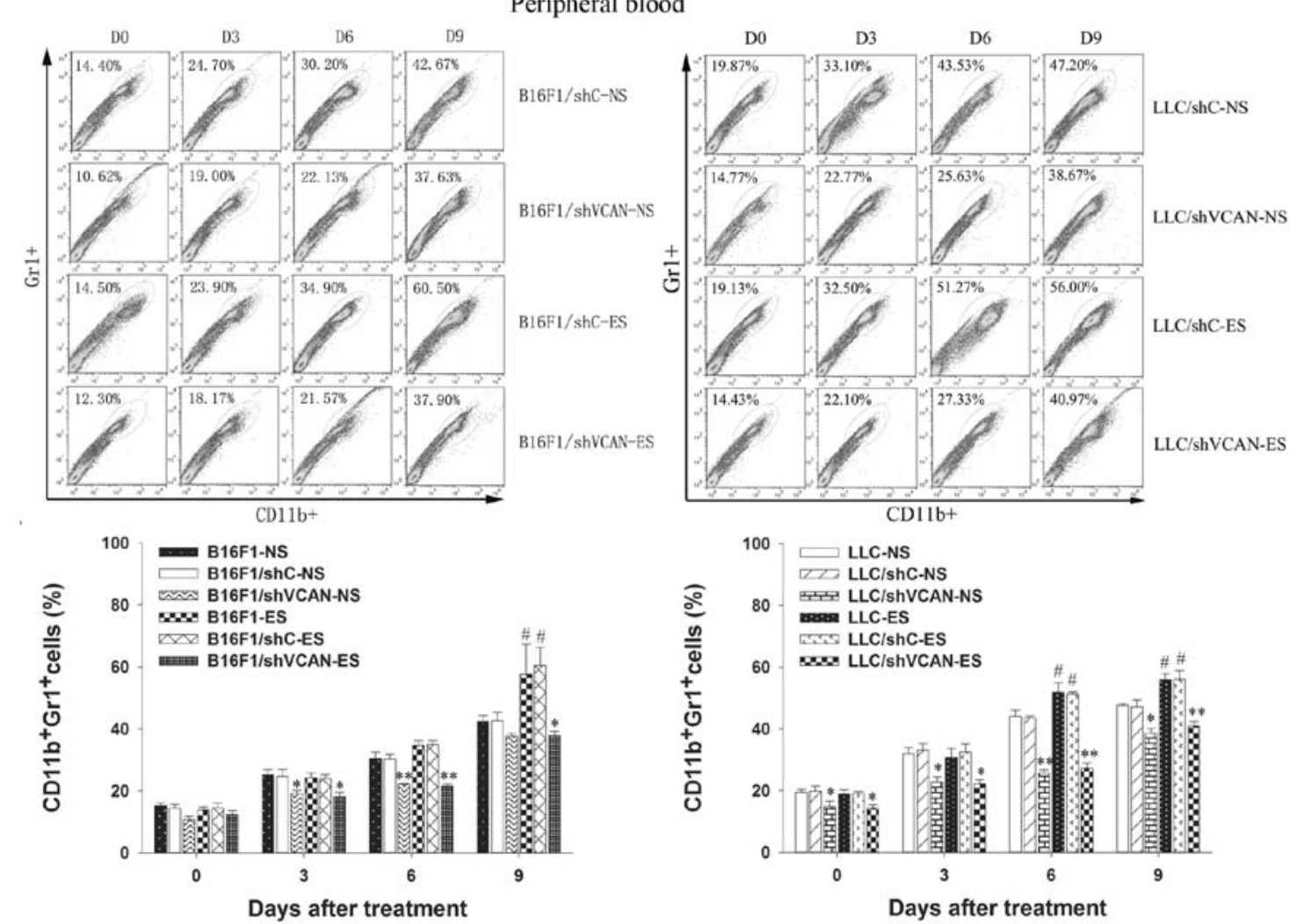

Figure 4. Versican silencing reduces the accumulation of MDSCs elicited by endostatin in peripheral blood. On days $0,3,6$ and 9 after the initiation of endostatin treatment, MDSCs in the B16F1 and LLC peripheral blood were detected by flow cytometry. $\mathrm{n}=3$. shVCAN vs. shC, ${ }^{*} \mathrm{P}<0.05$, ${ }^{* *} \mathrm{P}<0.01$. ES vs. NS, ${ }^{\#} \mathrm{P}<0.05,{ }^{\# \prime \prime} \mathrm{P}<0.01$. MDSCs, myeloid-derived suppressor cells; shVCAN, short-hairpin RNA targeting versican. 

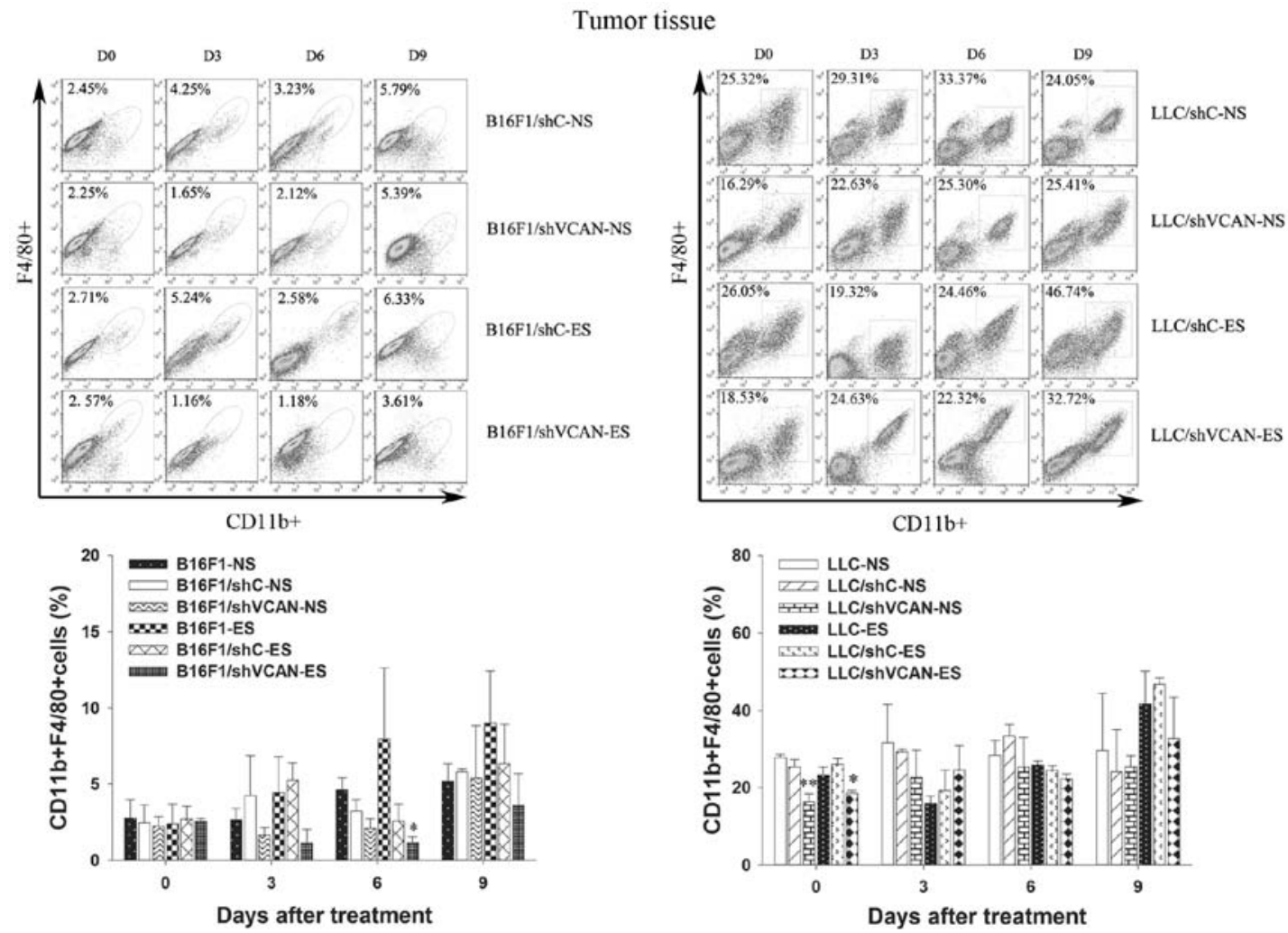

Figure 5. Versican silencing affects the presence of TAMs after endostatin treatment in tumor tissue. On days 0, 3, 6 and 9 after initiation of endostatin treatment, TAMs in the B16F1 and LLC tumor tissue were detected by flow cytometry; $\mathrm{n}=3$. shVCAN vs. shC, ${ }^{*} \mathrm{P}<0.05,{ }^{* *} \mathrm{P}<0.01$. TAMs, tumor-associated macrophages; LLC, Lewis lung carcinoma; shVCAN, short-hairpin RNA targeting versican.

including G-CSF, TNF- $\alpha$, IL-6, VEGF and IL-10. Fig. 6 shows the levels of these cytokines in the tumor tissue. On day 9 after treatment, a clear increase in G-CSF, TNF- $\alpha$, IL-6, VEGF and IL-10 was found in the endostatin monotherapy groups, as compared with the NS-treated models. However, a clear reduction in these cytokines was observed in all the shVCAN-ES groups, as compared with the endostatin monotherapy groups. Fig. 7 shows the levels of the aforementioned cytokines in the serum. On day 9 after treatment, a clear increase in G-CSF and VEGF was found in the endostatin monotherapy groups, as compared with the NS-treated groups. However, a clear reduction was observed in all the shVCAN-ES groups, as compared with the endostatin monotherapy groups (the same change in TNF- $\alpha$ was found on day 6 after treatment, in IL- 6 the change was identified on day 6-9 after treatment, and in IL-10 on day 3 and 9 after treatment).

The results indicated that endostatin elicited an increase in G-CSF, TNF- $\alpha$ and IL- 6 in serum and tumor tissue (more significant on day 9 after treatment), and to some extent versican silencing reversed this tendency. The level of VEGF decreased at an early stage of endostatin treatment (on day 3-6 after treatment), but reverted quickly on day 9 after treatment. The level of IL-10 in the serum decreased slightly at an early stage of endostatin treatment (on day 3-6 after treatment); however, it increased significantly on day 9 after treatment. Versican silencing alleviated the increase in IL-10 elicited by endostatin.

Versican silencing alleviated the endostatin-elicited tumor inflammatory microenvironment by leading to downregulation of $N F-\kappa B$ and $H I F-1 \alpha$. Versican and NF- $\kappa \mathrm{B}$ are generally acknowledged to be proinflammatory factors $(8,17)$. In order to explore the related mechanisms of how versican silencing alleviated the endostatin-elicited tumor inflammatory microenvironment, western blot analysis and immunohistochemical assays were conducted to determine the expression of versican, $\mathrm{NF}-\kappa \mathrm{B}$ and HIF-1 $\alpha$, and immunohistochemical assays were used to evaluate the expression of CD31 in tumor tissue.

The results showed that the expression of versican was attenuated in the shVCAN-transfected groups, as compared with the shC stable transfected and untransfected groups (Figs. 8A and 10), and that endostatin has the ability to increase the expression level of versican (Fig. 8A). With the duration of endostatin treatment, $\mathrm{NF}-\kappa \mathrm{B}$ increased gradually, but was reduced with versican silencing (Fig. $8 \mathrm{~B}$ and 10). Notably, on day 9 after treatment, a lower expression level of $\mathrm{NF}-\kappa \mathrm{B}$ was detected in the shVCAN+ES group compared with the shVCAN+NS group in the B16F1 tumors (Fig. 8B). With the duration of endostatin treatment, HIF-1 $\alpha$ increased gradually, but was reduced with versican silencing (Figs. 9A and 10). At an early stage of endostatin treatment (on day 3-6 after treatment), microvessel density was lower in the endostatin-treated group than in the NS-treated group ( $\mathrm{P}>0.05)$ (Fig. 9B). Compared with the endostatin monotherapy groups, the microvessel density decreased in the shVCAN+ES groups (in B16F1 tumor models, $\mathrm{P}<0.05$ ) (Fig. 9B).

\section{Discussion}

In the present study, B16F1 and LLC cell lines with stably silenced expression of versican were initially established, and 

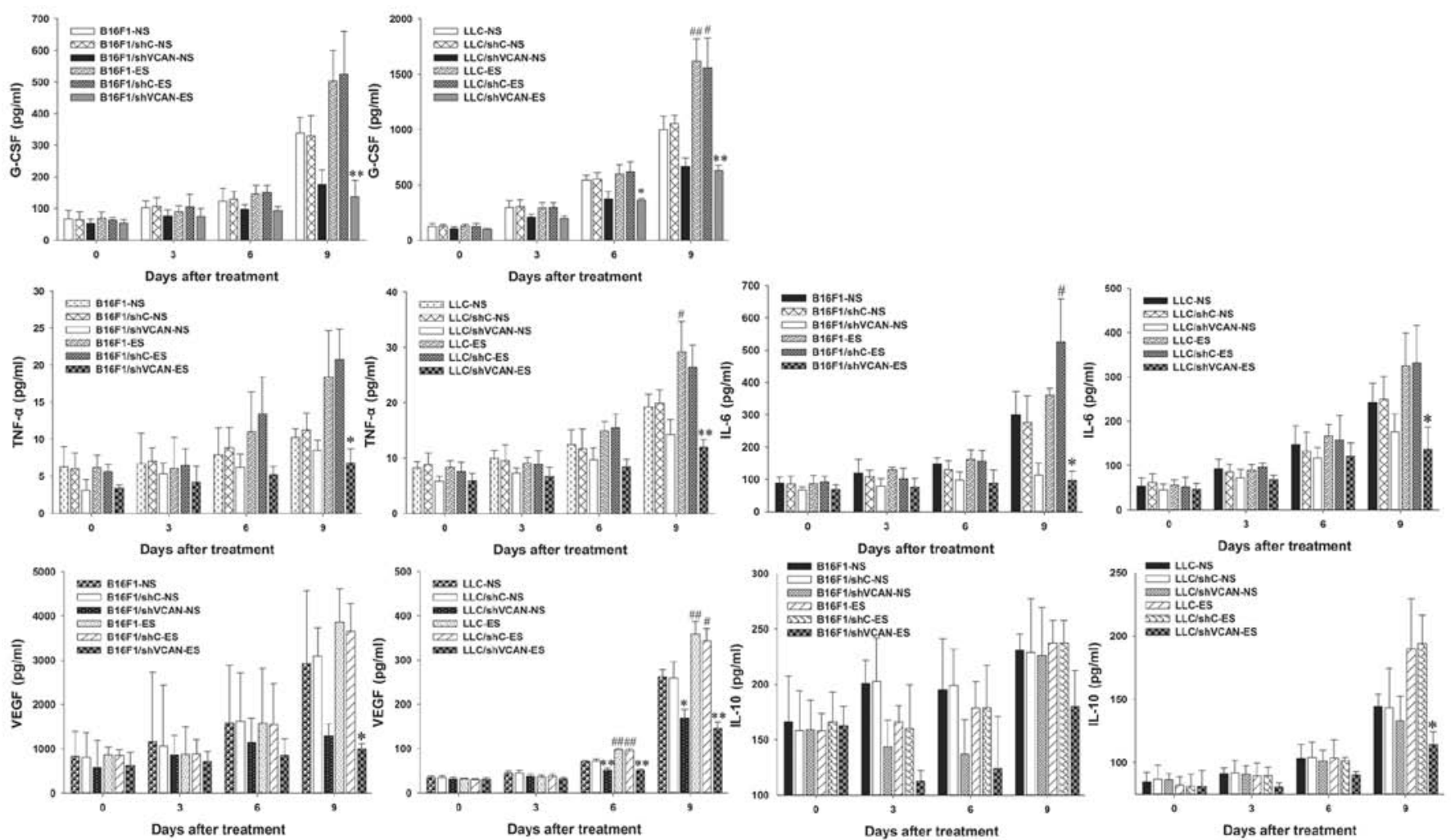

Figure 6. Versican silencing reduces the accumulation of tumor-associated inflammatory cytokines elicited by endostatin in tumor tissue. On days $0,3,6$ and 9 after the initiation of endostatin treatment, Luminex xMAP assays were conducted to determine the level of G-CSF, TNF- $\alpha$, IL-6, VEGF and IL-10 in tumor tissue of B16F1 and LLC tumor-bearing mice. $n=3$. shVCAN vs. shC, ${ }^{*} \mathrm{P}<0.05,{ }^{* *} \mathrm{P}<0.01$. ES vs. NS, ${ }^{*} \mathrm{P}<0.05,{ }^{\# \#} \mathrm{P}<0.01$. G-CSF, granulocyte colony-stimulating factor; TNF- $\alpha$, tumor necrosis factor- $\alpha$; VEGF, vascular endothelial growth factor; LLC, Lewis lung carcinoma; shVCAN, short-hairpin RNA targeting versican.
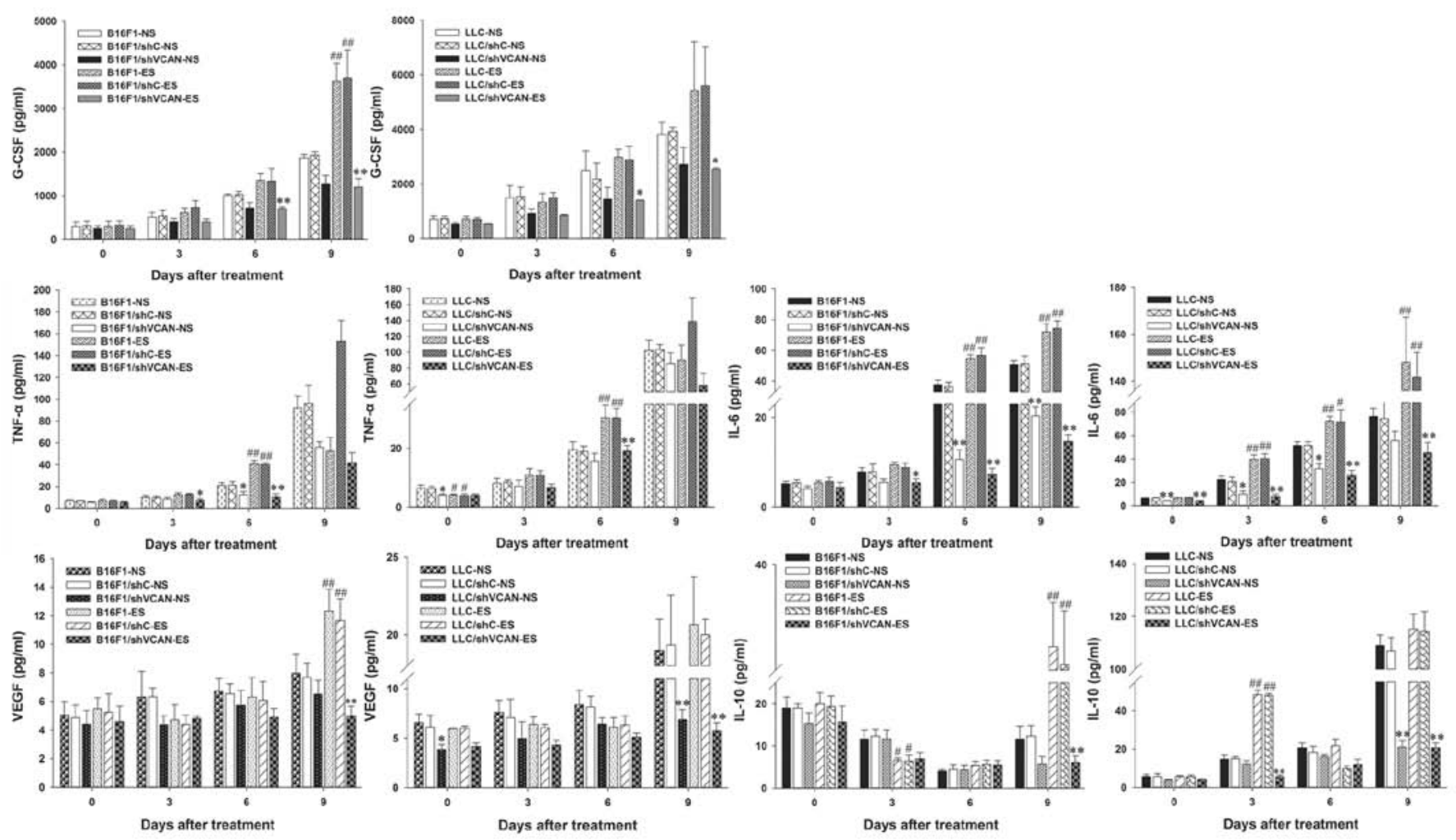

Figure 7. Versican silencing reduces the accumulation of tumor-associated inflammatory cytokines elicited by endostatin in serum. On days $0,3,6$ and 9 after the initiation of endostatin treatment, Luminex xMAP assays were conducted to determine the level of G-CSF, TNF- $\alpha$, IL-6, VEGF and IL-10 in the serum of B16F1 and LLC tumor-bearing mice. $\mathrm{n}=3$. shVCAN vs. shC, ${ }^{*} \mathrm{P}<0.05,{ }^{* *} \mathrm{P}<0.01$. ES vs. NS, ${ }^{*} \mathrm{P}<0.05,{ }^{\# \#} \mathrm{P}<0.01$. G-CSF, granulocyte colony-stimulating factor; TNF- $\alpha$, tumor necrosis factor- $\alpha$; VEGF, vascular endothelial growth factor; LLC, Lewis lung carcinoma; shVCAN, short-hairpin RNA targeting versican. 

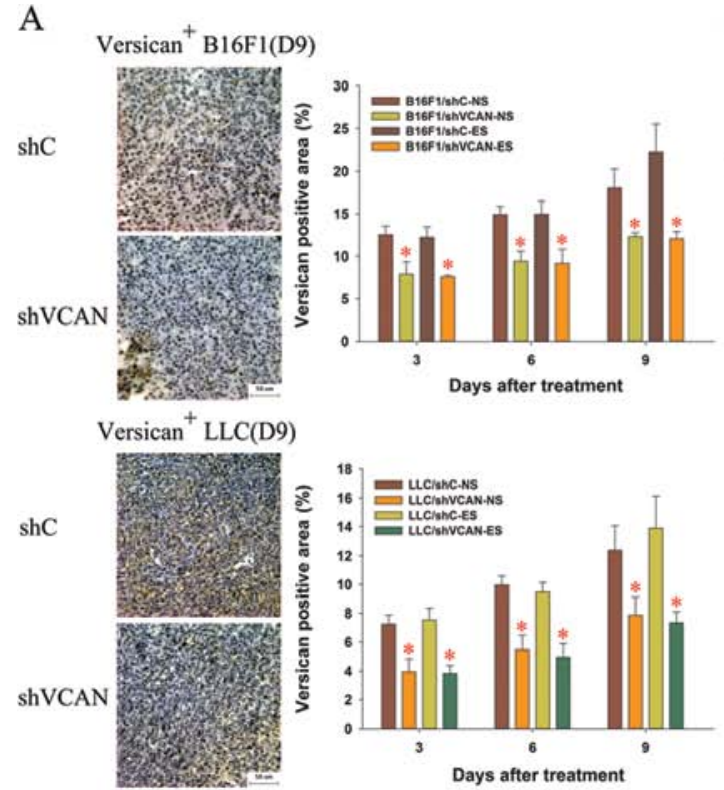

B

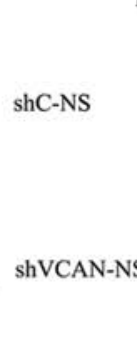

ShC-ES

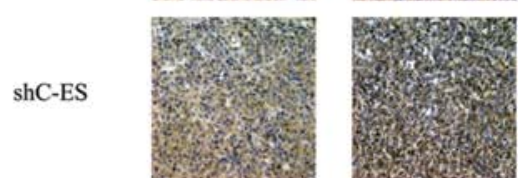

$\mathrm{NF}-\mathrm{KB}^{+} \mathrm{B} 16 \mathrm{~F} 1(\mathrm{D} 9) \mathrm{NF}-\mathrm{kB}{ }^{+}$LLC(D9)
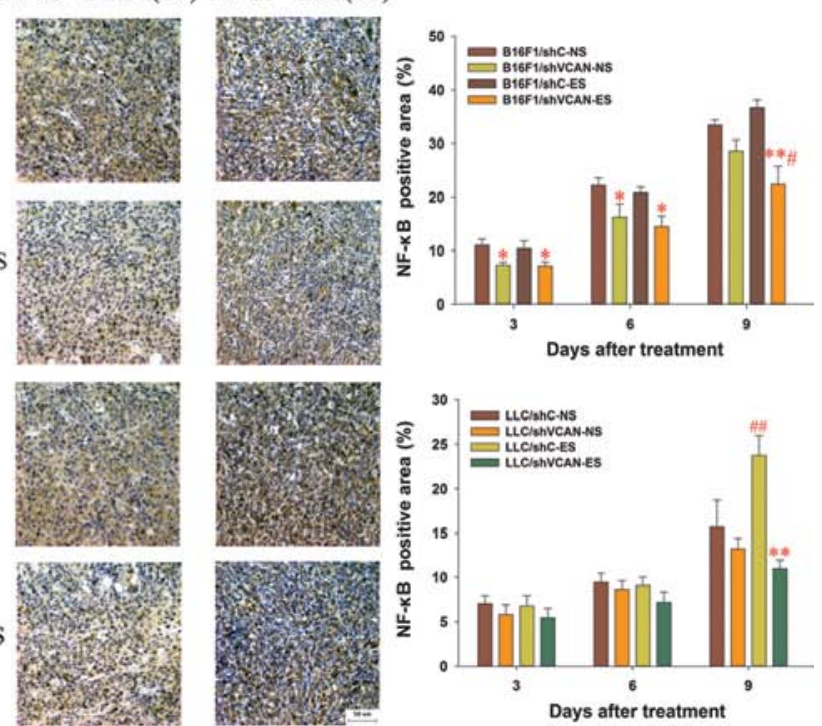

Figure 8. Immunohistochemical assays of the expression of versican and NF- $\mathrm{B}$ in B16F1 and LLC tumor tissue. On days 3 , 6 and 9 after the initiation of endostatin treatment, the expression of (A) versican and (B) NF- $\kappa \mathrm{B}$ was detected by immunohistochemical assays. Scale bar, $50 \mu \mathrm{m}$. Original magnification, $\mathrm{x} 400$ for all panels with a microscope. At least three random fields were evaluated for each section. shVCAN vs. shC, ${ }^{*} \mathrm{P}<0.05,{ }^{* *} \mathrm{P}<0.01$. ES vs. NS, ${ }^{\#} \mathrm{P}<0.05$, ${ }^{\#} \mathrm{P}<0.01$. LLC, Lewis lung carcinoma; shVCAN, short-hairpin RNA targeting versican.

A

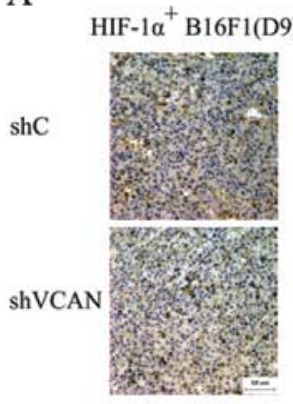

HIF-1 $\alpha^{+}$LLC(D9)

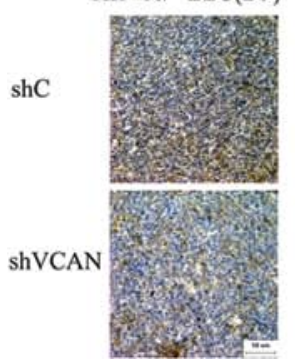

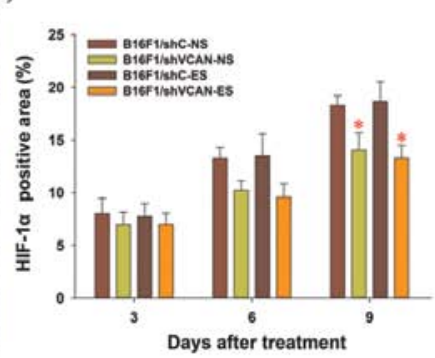

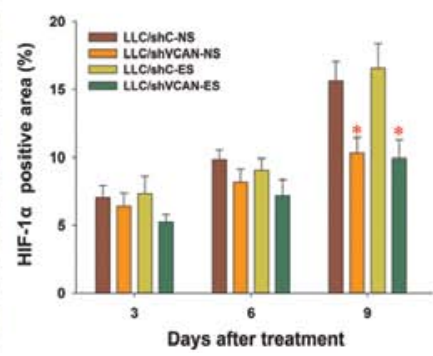

B
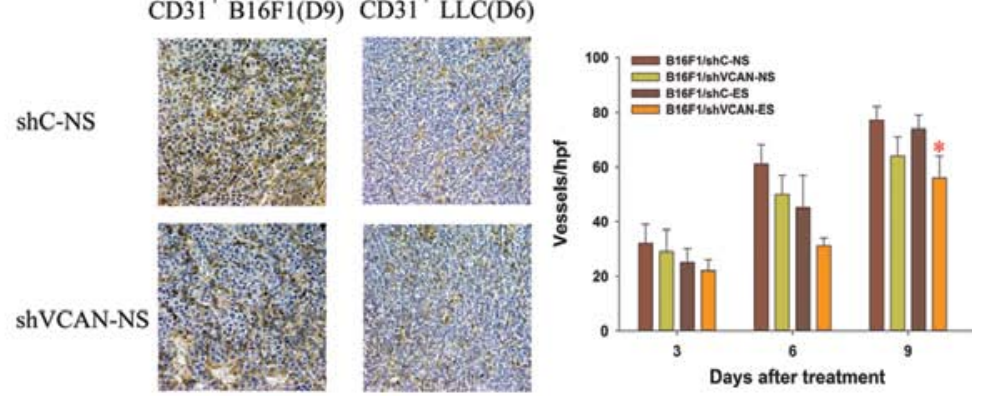

shC-ES
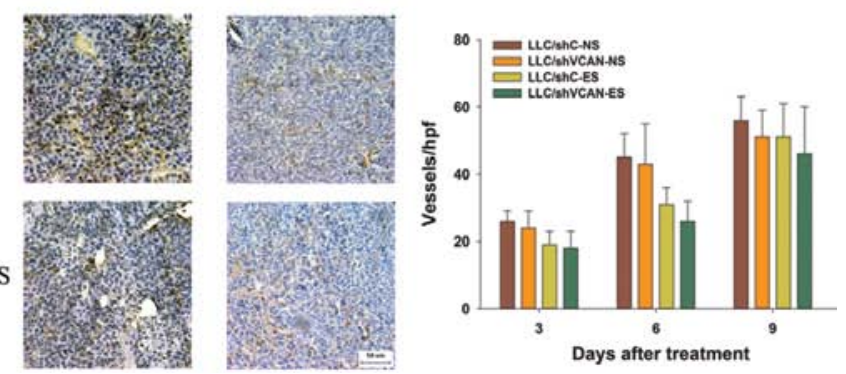

Figure 9. Immunohistochemical assays of the expression of HIF-1 $\alpha$ and CD31 in B16F1 and LLC tumor tissue. On days 3, 6 and 9 after the initiation of endostatin treatment, the expression of (A) HIF-1 $\alpha$ and (B) CD31 was detected by immunohistochemical assays. Scale bar, $50 \mu \mathrm{m}$. Original magnification, $\mathrm{x} 400$ for all panels with a microscope. At least three random fields were evaluated for each section. shVCAN vs. shC, ${ }^{*} \mathrm{P}<0.05,{ }^{* *} \mathrm{P}<0.01$. LLC, Lewis lung carcinoma; shVCAN, short-hairpin RNA targeting versican.

the effects of versican silencing on the bioactivity of shVCAN stable-transfected cell lines were assessed to eliminate its influence on exploring the role of versican in the development of th einflammatory tumor microenvironment in vivo. We found that versican silencing exerted an inhibitory effect on the proliferative and migratory ability of B16F1 cells, but did not affect LLC cells. Consistent with our findings, a previous study showed that versican V0/V1 silencing caused a reduction in the proliferation and migratory ability in human melanoma SK-mel-131 cell lines (18). Notably, the knockdown of versican expression in A549 lung cancer cells by RNA interference significantly inhibited tumor growth in vivo but not in vitro (19). Although versican has different effects on biological behavior in different cell lines its mechanisms remain to be elucidated.

Several observations have been made in the present study concerning combination therapy based on versican silencing and endostatin anti-angiogenesis. Endostatin showed modest 


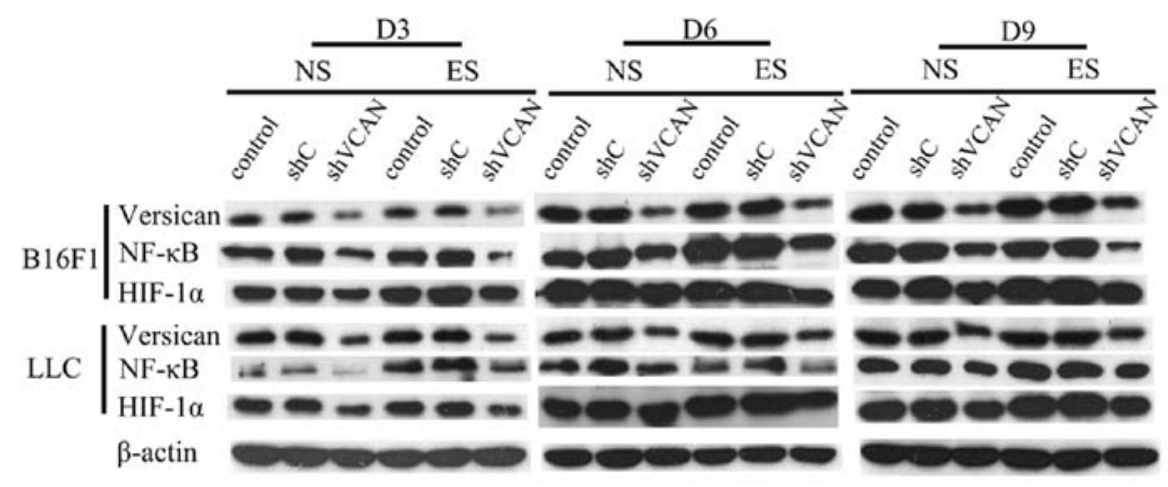

Figure 10. Western blot analysis of the expression of versican, NF- $\mathrm{kB}$ and HIF-1 $\alpha$ in B16F1 and LLC tumor tissue. On days 3, 6 and 9 after the initiation of endostatin treatment, the expression of versican, NF- $\mathrm{KB}$ and HIF-1 $\alpha$ was determined using western blot analysis. The internal reference was presented by $\beta$-actin. LLC, Lewis lung carcinoma.

inhibition of tumor growth, and had no effect on the survival time of tumor-bearing mice. Versican silencing alone effectively suppressed orthotopic tumor growth and significantly prolonged survival time in the B16F1 and LLC tumor models. This effect was enhanced with combined versican silencing and endostatin treatment. The major cause of the tumor refractoriness to the antiangiogenic therapy may be associated with the inflammatory and immunosuppressive changes of the tumor microenvironment elicited by endostatin administration. By reducing the recruitment of tumor-associated inflammatory and immunosuppressive cells, and achieving remission of tumor-inflammatory cytokines, versican silencing of cancer cells in the tumor microenvironment alleviated the tumor refractoriness to antiangiogenic therapy with endostain. These suggestions were supported by our own results. Myeloidderived suppressor cells (MDSCs) and tumor-associated macrophages (TAMs) in the tumor tissue and peripheral blood of B16F1 and LLC tumor-bearing mice were examined by flow cytometry, and the inflammatory cytokines (including G-CSF, TNF- $\alpha$, IL-6, VEGF and IL-10) were assessed by Luminex xMAP assays. A clear increase in MDSCs in the blood and tumor tissue was identified in all the endostatintreated groups of tumor-bearing models, as compared with NS-treated ones (more significant on day 9 after treatment). However, the versican silencing of cancer cells reversed this tendency. On day 9 after treatment, an increase in TAMs was found in the tumor tissue in all the endostatin-treated groups of tumor-bearing models $(\mathrm{P}>0.05)$. Versican silencing reduced the number of TAMs in the tumor tissue on day 6 after treatment in $\mathrm{B} 16 \mathrm{~F} 1$ tumors, and on day 0 after treatment in LLC tumors. In addition, endostatin caused an increase in G-CSF, TNF- $\alpha$ and IL- 6 in serum and tumor tissue (more significant on day 9 after treatment), and to some extent, versican silencing reversed this tendency. There was a reduced level of VEGF and IL-10 in serum and tumor tissue at the early stage of endostatin treatment (on days 3-6 after treatment). However, the level of VEGF and IL-10 increased quickly on day 9 after the treatment. Versican silencing attenutated the increase in IL-10 elicited by endostatin.

$\mathrm{CD}_{11 \mathrm{~b}^{+} \mathrm{Grl}}{ }^{+}$MDSCs, a class of immature myeloid cells, also described as a subset of tumor-associated inflammatory cells, are recruited by inflammation cytokines secreted by tumor and stromal cells (20). It is a commonly held view that
MDSCs act as 'bridges' linking inflammation and cancer (20). MDSCs are induced by tumor-secreted and host-secreted factors, many of which are proinflammatory molecules, such as TGF- $\beta$, TNF- $\alpha$, IL- 6 , IL-10, G-CSF, GM-CSF, CCL2 and CXCL12 $(20,21)$. The induction of MDSCs by proinflammatory mediators led to the hypothesis that inflammation promotes the accumulation of MDSCs which in turn, downregulate immune surveillance and antitumor immunity, thereby facilitating tumor growth (20). In addition, MDSCs play an important role in tumor growth through the induction of angiogenesis (21). Previous findings have suggested that MDSCs contribute to refractoriness to antiangiogenic therapy, and that the recruitment of MDSCs stimulated by overexpressed proinflammatory factors after anti-VEGF treatment is a major cause of the resistance of tumors to antiangiogenic therapies $(22,23)$. In the present study, we found that antiangiogenic therapy with endostatin elicited inflammatory and immunosuppressive changes in the tumor microenvironment, including the accumulation of MDSCs and TAMs, and an increase in G-CSF, TNF- $\alpha$, IL-6, VEGF and IL-10. The versican silencing alleviated the inflammatory and immunosuppressive changes in the tumor microenvironment, and improved the antitumor efficacy of endostatin. Our findings may contribute in elucidating the inflammation promoting the accumulation of MDSCs, which in turn, facilitate tumor progression.

TAMs comprise a heterogeneous cell population originating from mononuclear phagocytic lineage $(24,25)$. TAMs are the second well-described population of myeloid cells that have been shown to exert a negative effect on antitumor immune responses. The relationship between TAMs and MDSCs has not been completely defined, although it has been suggested that TAMs may in part be derived from or be associated with MDSCs (26). Diversity and plasticity are characteristics of TAMs, which can be polarized to different phenotypic subgroups under different microenvironmental conditions (27). The classically activated (M1-like) TAMs reduce angiogenesis, increase inflammation and express antitumor activity. The mediators (IL-12, TNF- $\alpha$ and IL-6) of M1 macrophage-mediated inflammation maintain a high level by M1 macrophage (28). However, the alternatively activated (M2-like) TAMs usually promote tumor growth and stimulate angiogenesis $(29,30)$. M2 macrophages exhibit immune suppression through the production of immunosuppressive 
cytokines (e.g., IL-10 and TGF- $\beta$ ) and the recruitment of regulatory $\mathrm{T}$ cells through the secretion of CCL22 (28). In the tumor microenvironment, many cytokines skew the polarization of TAMs from an M1-like phenotype to an M2-like phenotype (e.g., IL-4, IL-6, IL-13, IL-10 and TGF- $\beta$ ) (31). In general, most TAMs in the tumor microenvironment obtain M2-like properties and resemble 'tolerant' macrophages, and an increased number of TAMs correlate with vessel density and poor prognosis (30). In the present study, we found that TAMs in the tumor microenvironment increased modestly on day 9 after endostatin treatment $(\mathrm{P}>0.05)$. Versican silencing reduced the number of TAMs in the tumor tissue on day 6 after treatment in B16F1 tumors, and on day 0 after treatment in LLC tumors. However, versican silencing had no effect on TAMs on day 9 after endostatin treatment, possibly caused by the polarization of TAMs from an M2-like phenotype to an M1-like phenotype. We also found that IL-6 and IL-10 in the tumor microenvironment and serum, which skewed the polarization of TAMs from an M1-like to an M2-like phenotype, increased with endostatin treatment but were reduced by versican silencing. Moreover, the level of TNF- $\alpha$ and VEGF, which can be secreted by M2 macrophages, were also downregulated by versican silencing. These findings indicate that versican silencing can skew the polarization of TAMs from an M2-like phenotype to an M1-like phenotype through the regulation of the cytokines described above.

Among the many different mediators of the recruitment of inflammatory cells into the tumor microenvironment, versican and NF- $\mathrm{kB}$ are generally acknowledged to be proinflammatory factors $(8,17)$. Versican strongly enhances tumor progression by activating multiple types of inflammatory cells by combining with the cell surface of TLR-2, and eliciting the production of proinflammatory cytokines (8). The inflammatory cytokines (e.g., TNF- $\alpha$, IL-1 $\beta$, IL- 6 and IL-8) are involved in the TLR-2-mediated NF- $\kappa$ B pathway, which regulates the transcription genes associated with the immune and inflammatory responses (32). Recently, investigators reported that hemiterpene rotundarpene (4-caffeoyl-3-methyl-but-2-ene-1,4-diol, an extract from the bark of the Ilex rotunda Thunb.) attenuated the production of inflammatory mediators by suppressing activation of the TLR-2-mediated NF- $\kappa \mathrm{B}$ pathway (32). Although versican and NF- $\mathrm{\kappa B}$ are proinflammatory factors, the direct relationship between them remains elusive. In the present study, as previously described, we found that versican silencing alleviated the inflammatory and immunosuppressive changes elicited by endostatin, and disrupted the positive feedback for the extension of inflammation, and thus improved the antitumor efficacy of endostatin. In order to explore the associated mechanisms, we conducted immunohistochemical and western blot analyses to determine the expression of versican, NF- $\mathrm{KB}$ and HIF- $1 \alpha$. We found that the expression of NF- $\kappa B$ and HIF- $1 \alpha$ was greater in the endostatin monotherapy group compared with the NS-treated ones, but decreased in the shVCAN-transfected groups. This finding suggested that versican silencing resulted in the downregulation of NF- $\mathrm{KB}$ and HIF- $1 \alpha$. Versican affected the expression of NF- $\kappa B$ via many inflammatory cytokines, including G-CSF, TNF- $\alpha$ and IL-6, which were involved in the TLR-2-mediated NF- $\mathrm{KB}$ pathway. Using Luminex xMAP assays, we found that there was a clear increase in G-CSF, TNF- $\alpha$ and IL-6 in endostatin monotherapy groups in the B16F1 and LLC tumor models on day 9 after endostatin treatment, as compared with NS-treated ones. However, a clear reduction in G-CSF, TNF- $\alpha$ and IL-6 was observed in the shVCAN-transfected groups, as compared with the shC-transfected and untransfected ones. These findings indicate that versican silencing reduced the accumulation of G-CSF, TNF- $\alpha$ and IL- 6 elicited by endostatin in serum and tumor tissue. Based on the results, we hypothesize that the silencing of versican may suppress activation of the TLR-2mediated NF- $\mathrm{KB}$ pathway by reducing the production of inflammatory cytokines (e.g., TNF- $\alpha$ and IL-6) involved in this pathway. However, additional studies are required to clarify the exact mechanisms of the interaction of versican and NF- $\mathrm{\kappa B}$.

In summary, our findings indicate that the leading cause of tumor refractoriness to antiangiogenic therapy is associated with inflammatory and immunosuppressive changes in the tumor microenvironment elicited by endostatin administration. Versican silencing improved the antitumor efficacy of endostatin by alleviating its induced alterations in the tumor microenvironment. Versican silencing in the tumor microenvironment may offer a promising approach to reverse the tumor refractoriness to antiangiogenic therapies.

\section{Acknowledgements}

The present study funding was supported by the National Natural Science Foundation of China (nos. 81071864 and 81372506).

\section{References}

1. Verheul HM, Hammers H, van Erp K, Wei Y, Sanni T, Salumbides B, Qian DZ, Yancopoulos GD and Pili R: Vascular endothelial growth factor trap blocks tumor growth, metastasis formation, and vascular leakage in an orthotopic murine renal cell cancer model. Clin Cancer Res 13: 4201-4208, 2007.

2. Paez-Ribes M, Allen E, Hudock J, Takeda T, Okuyama H, Vinals F, Inoue M, Bergers G, Hanahan D and Casanovas O: Antiangiogenic therapy elicits malignant progression of tumors to increased local invasion and distant metastasis. Cancer Cell 15: 220-231, 2009.

3. Zuniga RM, Torcuator R, Jain R, Anderson J, Doyle T, Ellika S, Schultz L and Mikkelsen T: Efficacy, safety and patterns of response and recurrence in patients with recurrent highgrade gliomas treated with bevacizumab plus irinotecan. J Neurooncol 91: 329-336, 2009.

4. Shojaei F, Wu X, Malik AK, Zhong C, Baldwin ME, Schanz S, Fuh G, Gerber HP and Ferrara N: Tumor refractoriness to anti-VEGF treatment is mediated by $\mathrm{CD} 11 \mathrm{~b}^{+} \mathrm{Grl}^{+}$myeloid cells Nat Biotechnol 25: 911-920, 2007.

5. Soeda S, Nakamura N, Ozeki T, Nishiyama H, Hojo H, Yamada H, Abe M and Sato A: Tumor-associated macrophages correlate with vascular space invasion and myometrial invasion in endometrial carcinoma. Gynecol Oncol 109: 122-128, 2008.

6. Kischel P, Waltregny D, Dumont B, Turtoi A, Greffe Y, Kirsch S, De Pauw E and Castronovo V: Versican overexpression in human breast cancer lesions: Known and new isoforms for stromal tumor targeting. Int J Cancer 126: 640-650, 2010.

7. Wang W, Xu GL, Jia WD, Ma JL, Li JS, Ge YS, Ren WH, Yu JH and Liu WB: Ligation of TLR2 by versican: A link between inflammation and metastasis. Arch Med Res 40: 321-323, 2009.

8. Kim S, Takahashi H, Lin WW, Descargues P, Grivennikov S, Kim Y, Luo JL and Karin M: Carcinoma-produced factors activate myeloid cells through TLR2 to stimulate metastasis. Nature 457: 102-106, 2009.

9. Gao D, Joshi N, Choi H, Ryu S, Hahn M, Catena R, Sadik H, Argani P, Wagner P, Vahdat LT, et al: Myeloid progenitor cells in the premetastatic lung promote metastases by inducing mesenchymal to epithelial transition. Cancer Res 72: 1384-1394, 2012. 
10. Ling Y, Yang Y, Lu N, You QD, Wang S, Gao Y, Chen Y and Guo QL: Endostar, a novel recombinant human endostatin, exerts antiangiogenic effect via blocking VEGF-induced tyrosine phosphorylation of KDR/Flk-1 of endothelial cells. Biochem Biophys Res Commun 361: 79-84, 2007.

11. Wang HL, Ning T, Li M, Lu ZJ, Yan X, Peng Q, Lei N, Zhang H and Luo F: Effect of endostatin on preventing postoperative progression of distant metastasis in a murine lung cancer model. Tumori 97: 787-793, 2011.

12. Ning T, Yan X, Lu ZJ, Wang GP, Zhang NG, Yang JL, Jiang SS Wu Y, Yang L, Guan YS, et al: Gene therapy with the angiogenesis inhibitor endostatin in an orthotopic lung cancer murine model. Hum Gene Ther 20: 103-111, 2009.

13. Ning T, Jiang M, Peng Q, Xi Y, Lu ZJ, Peng YL, Wang HL, Lei N, Zhang $\mathrm{H}$, Lin HJ, et al: Low-dose endostatin normalizes the structure and function of tumor vasculature and improves the delivery and anti-tumor efficacy of cytotoxic drugs in a lung cancer xenograft murine model. Thorac Cancer 3: 229-238, 2012.

14. Huang G and Chen L: Discrepancies between antiangiogenic and antitumor effects of recombinant human endostatin. Cancer Biother Radiopharm 24: 589-596, 2009.

15. Zhang H, Wang Z, Peng Q, Liu YY, Zhang W, Wu L, Wang X and Luo F: Tumor refractoriness to endostatin anti-angiogenesis is associated with the recruitment of $\mathrm{CD} 11 \mathrm{~b}+\mathrm{Gr} 1+$ myeloid cells and inflammatory cytokines. Tumori 99: 723-733, 2013.

16. Li XQ, Shang BY, Wang DC, Zhang SH, Wu SY and Zhen YS: Endostar, a modified recombinant human endostatin, exhibits synergistic effects with dexamethasone on angiogenesis and hepatoma growth. Cancer Lett 301: 212-220, 2011.

17. Ben-Neriah Y and Karin M: Inflammation meets cancer, with $\mathrm{NF}-\kappa \mathrm{B}$ as the matchmaker. Nat Immunol 12: 715-723, 2011.

18. Hernández D, Miquel-Serra L, Docampo MJ, Marco-Ramell A and Bassols A: Role of versican V0/V1 and CD44 in the regulation of human melanoma cell behavior. Int J Mol Med 27: 269-275, 2011

19. Zheng PS, Wen J, Ang LC, Sheng W, Viloria-Petit A, Wang Y, Wu Y, Kerbel RS and Yang BB: Versican/PG-M G3 domain promotes tumor growth and angiogenesis. FASEB J 18: 754-756, 2004.

20. Ostrand-Rosenberg S and Sinha P: Myeloid-derived suppressor cells: Linking inflammation and cancer. J Immunol 182: 4499-4506, 2009.
21. Gabrilovich DI and Nagaraj S: Myeloid-derived suppressor cells as regulators of the immune system. Nat Rev Immunol 9: $162-174,2009$

22. Shojaei F, Wu X, Qu X, Kowanetz M, Yu L, Tan M, Meng YG and Ferrara N: G-CSF-initiated myeloid cell mobilization and angiogenesis mediate tumor refractoriness to anti-VEGF therapy in mouse models. Proc Natl Acad Sci USA 106: 6742-6747, 2009.

23. Carbone C, Moccia T, Zhu C, Paradiso G, Budillon A, Chiao PJ, Abbruzzese JL and Melisi D: Anti-VEGF treatment-resistant pancreatic cancers secrete proinflammatory factors that contribute to malignant progression by inducing an EMT cell phenotype. Clin Cancer Res 17: 5822-5832, 2011.

24. Gordon S and Taylor PR: Monocyte and macrophage heterogeneity. Nat Rev Immunol 5: 953-964, 2005.

25. Pollard JW: Trophic macrophages in development and disease. Nat Rev Immunol 9: 259-270, 2009.

26. Sica A and Bronte V: Altered macrophage differentiation and immune dysfunction in tumor development. J Clin Invest 117: 1155-1166, 2007.

27. Martinez FO, Helming L and Gordon S: Alternative activation of macrophages: An immunologic functional perspective. Annu Rev Immunol 27: 451-483, 2009.

28. Mantovani A, Bottazzi B, Colotta F, Sozzani S and Ruco L: The origin and function of tumor-associated macrophages. Immunol Today 13: 265-270, 1992.

29. Mantovani A and Sica A: Macrophages, innate immunity and cancer: Balance, tolerance, and diversity. Curr Opin Immunol 22: 231-237, 2010.

30. Qian BZ and Pollard JW: Macrophage diversity enhances tumor progression and metastasis. Cell 141: 39-51, 2010.

31. Sica A, Saccani A and Mantovani A: Tumor-associated macrophages: A molecular perspective. Int Immunopharmacol 2: 1045-1054, 2002

32. Kim YJ, Jung EB, Lee MS, Seo SJ, Kim MH, Lee MW and Lee CS: Rotundarpene inhibits toll-like receptor 2 activationinduced production of inflammatory mediators in keratinocytes by suppressing the Akt and NF- $\kappa$ B pathways. Int Immunopharmacol 18: 325-332, 2014 